$\$=-$ 国

\title{
Analysis of operating noise characteristics and contribution for automotive seat backrest adjuster
}

\author{
Sung-Yuk Kim ${ }^{1 *}$, Key-Sun Kim ${ }^{2}$ \\ ${ }^{1}$ Department of Mechanical Engineering, Graduate School, Kongju National University, 1223-24, Cheonan-daero, Seobuk-gu, \\ Cheonan-si, Chungcheongnam-do, 31080, Republic of Korea \\ ${ }^{2}$ Division of Mechanical \& Automotive Engineering, Kongju National University, 1223-24, Cheonan-daero, Seobuk-gu, Cheonan-si, \\ Chungcheongnam-do, 31080, Republic of Korea \\ *Corresponding author E-mail: keysun@kongju.ac.kr
}

\begin{abstract}
Background/Objectives: This study involved basic research on operating noise in an automotive seat backrest adjuster through analysis of noise sources, noise source contributions, and seat pad noise reduction effects.

Methods/Statistical analysis: The test product was a driver seat of 3300cc commercial sedan. First, basic data is obtained through analysis of driving mechanism of seat backrest adjuster. Based on this, vibration and noise characteristics were analyzed by operating test of backrest adjuster, and contribution analysis to noise sources was performed. Finally, by analyzing the effect of noise reduction on the seat pad, the effect of each noise source on the backrest operating noise was determined.

Findings: First, it was confirmed that the low frequency fluctuation caused by the total noise is dependent on change in the load generated by eccentric rotation of the recliner gear. Therefore, it was found that the design of the recliner gear teeth was a decisive factor in controlling the motor load. Second, the 20th order $(1,320 \mathrm{~Hz})$ of the DC geared motor was the main cause of operating noise, which was derived from friction noise of brush-commutator and torque ripple noise. This noise accounted for more than $55 \%$ of total operating noise. In addition, the 30th order amounted to approximately $18 \%$. In other words, the harmonic components of the DC geared motor created most of the noise, and changing the brush material and reducing torque ripple is expected to significantly improve the overall operating noise. Lastly, as a result of the seat pad noise reduction effect, total power decreased by more than $11 \mathrm{~dB}$ (A). In addition, it was confirmed that the 20th order cause of the main noise was reduced by about $45 \%$ p.

Improvements/Applications: The noise contribution of the seat recliner adjuster derived from this study can be used as a basic data for noise reduction.
\end{abstract}

Keywords: Automotive; Power Seat; Backrest Adjuster; Operating Noise; Contribution.

\section{Introduction}

In recent years, consumer demand for convenience and comfort in automotive seats has been increasing[1,2]. Power seats have been developed for the general purpose of providing convenient functions using a small-sized motor and, recently, attention has been focused on providing a high-sensitivity seat that can simultaneously satisfy both drivers' convenience and comfort demands [3], [4] However, these convenient functions are based on complicated mechanisms, which cause vibration and noise to be generated during operation, and this has emerged as an important issue to be addressed. Of particular interest is the backrest adjuster, which is a device that adjusts the back angle of the seat and is one of the most heavily used seat devices. The backrest adjuster is typically composed of a recliner and a DC geared motor, each of which is a major cause of operating noise and vibration [5]. In the case of the recliner, it usually consists of a gear wheel, gear rim, cam, rod, wedge, dry bush, spring, cover, and housing [6], [7]. Here, the cause of operating noise from the recliner is the change in load due to the eccentric motion of the gear wheel, gear mesh frequency, and chattering. Of these, the gear wheel and gear rim are the most important factors in a steady-state drive because they have a significant influence on the load of the motor. In a DC geared motor, the friction noise of the brush-commutator, gear mesh frequency, and torque ripple are the main noise sources. The friction noise of the brushcommutator is influenced by the angle of the brush and the slot of the commutator. The torque ripple is most affected by the permanent magnet and core slot. All of these are harmonic components of the motor RPM frequency and all cause noise and vibration [8], [9]. In this way, various noise components in the backrest adjuster can be estimated via analysis of the driving mechanism. However, to date, satisfactory analysis of the influence of these noise components on the backrest adjuster has been lacking. As such, analysis of the contribution of the various noise sources should be prioritized in order ultimately to attempt to reduce this noise. In addition, the effect of the seat pad must be considered so as to accurately establish noise reduction measures. Therefore, this study involved basic research on operating noise in an automotive seat backrest adjuster through analysis of noise sources, noise source contributions, and seat pad noise reduction effects. First, basic data was obtained through analysis of the seat backrest adjuster driving mechanism. Based on this, vibration and noise characteristics were analyzed from a backrest adjuster operating test, and noise source contribution analysis was then performed. Finally, by analyzing the effect of noise reduction on the seat pad, the effect of each noise source on overall backrest operating noise was determined. 


\section{Test methodology}

Figure 1 shows the conditions for the operation test of the backrest adjuster and the sensor mounting position. The test product was the drivers' seat from a 3300cc commercial sedan. The test methodology was as follows: First, the reference position of the seat back was set at $90^{\circ}$ with respect to the headrest pole. Next, a load of $100 \mathrm{~N}$ was applied to the center of the seat back using a dummy. Third, the operating mode of the seat back consisted of a $42^{\circ}$ reclining movement from the reference position[10]. Fourth, environmental conditions were as follows: As shown in Figure 2, the test room was a semi-anechoic room with less than $30 \mathrm{~dB}$ (A) of ambient noise. Temperature was maintained at $20 \pm 2{ }^{\circ} \mathrm{C}$, and $30 \pm 10 \%$ humidity. Lastly, in terms of the location of the microphone and accelerometers, the microphone was installed $250 \mathrm{~mm}$ away from the headrest pole. And the accelerometers were attached to the DC-geared motor and the left and right recliner in the shaft axis direction. The measurement instruments and sensors used in this test were two NI DAQ 9218 (National Instruments Corporation), one 1/2 inch microphone (PCB Piezotronics Inc.), and three single axis accelerometers (PCB Piezotronics Inc.) as shown in Figure 2.
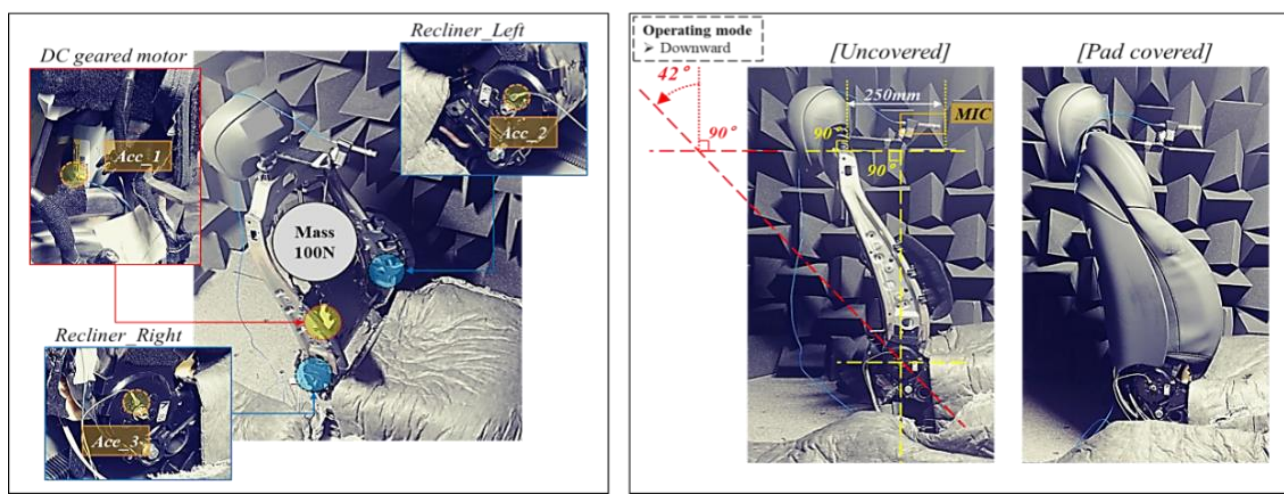

Fig. 1: Test Mode and Sensor Position.
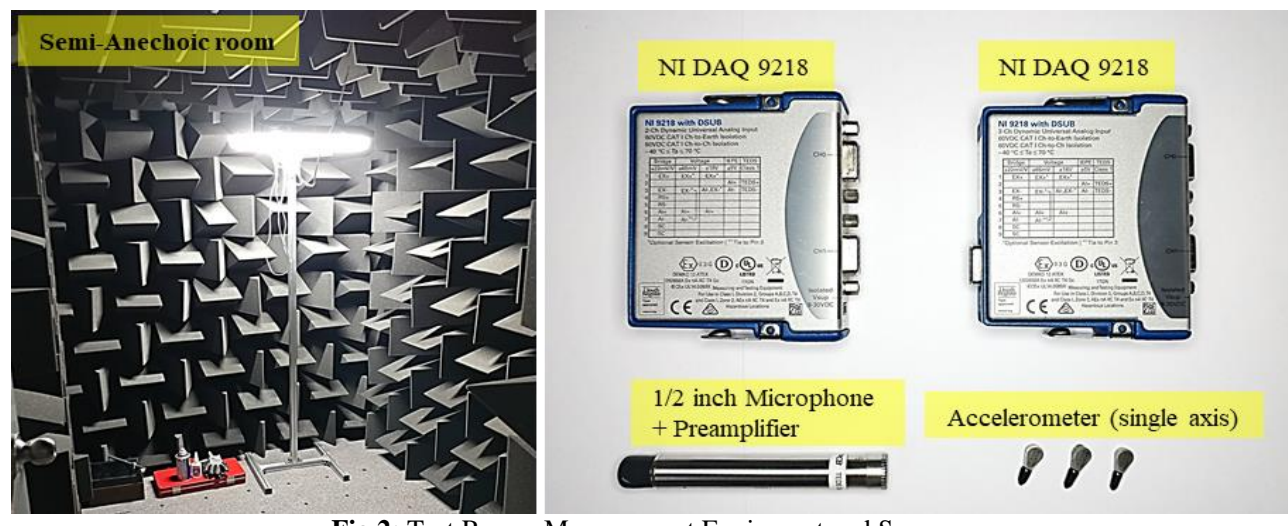

Fig 2: Test Room, Measurement Equipment and Sensors.

\section{Analysis and discussion}

\subsection{Analysis of the operating noise factor}

A DC geared motor consists of a brush, permanent magnet, commutator, laminated core, and reduction gear, as shown in Figure 3. There were three major noise generation factors: friction noise between the brush and commutator, torque ripple between the permanent magnet and coil, and gear mesh frequency from the reduction gear. First, the frequency of friction noise between the brush and the commutator depends on the angle of the brush and the number of commutator slots. That is, as shown in Fig. 4, as the commutator's slots passes through the brush, events occur that cause noise and vibration as harmonic components of the motor. Equation (1) shows the formula used for obtaining the frequency that occurs when two brushes have a phase of $90^{\circ}$.

$f_{\text {brush }}=\frac{r p m}{60} \times N_{\text {brush }} \times N_{\text {C.slot }}[\mathrm{Hz}]$ (N $\mathrm{N}_{\text {brush: }}$ number of brush, $\mathrm{N}_{\text {C.slot: number of commutator slots) }}$

In the case of torque ripple, when permanent magnets form a magnetic field and a current flow from the commutator to the coil, electromagnetic force is induced in accordance with Lorentz's law, causing rotation. Here, torque fluctuation occurs due to the distance between the coil and the permanent magnet, which is termed "torque ripple". Torque ripple is determined by the number of poles and the winding of the coil. A 4-pole, 10-slot motor was used in this study, and the torque ripple frequency was derived from Equation (2).

$f_{\text {torque }}=\frac{r p m}{60} \times 2 N_{\text {core }}[\mathrm{Hz}]\left(\mathrm{N}_{\text {core }}\right.$ number of laminated cores $)$

The reduction gear consisted of a worm and helical gear as shown in Figure 3. These gear meshes generate a gear mesh frequency, causing noise and vibration. Gear mesh frequency could be obtained using Equation (3).

$f_{G M F}=f_{r} \times N_{g}[\mathrm{~Hz}]\left(\mathrm{f}_{\mathrm{r}}\right.$ : frequency of rotation, $\mathrm{N}_{\mathrm{g}}$ : number of gear teeth)

Next, a recliner consists of eight parts shown in Figure 5, of which the gear rim and gear wheel form an internal gear, in that the eccentric motion of the gear wheel possesses a mechanism to directly control the movement of the seat back frame. The number of teeth of gear rim and gear wheel were 34 and 33, respectively. The seat back frame moved approximately $10.6^{\circ}$ per single revolution of the gear wheel. The gear mesh frequency of the recliner gear could again be calculated using Equation (3). Table 1 shows the frequency components of the DC geared motor and recliner derived from Equations (1) to (3). 


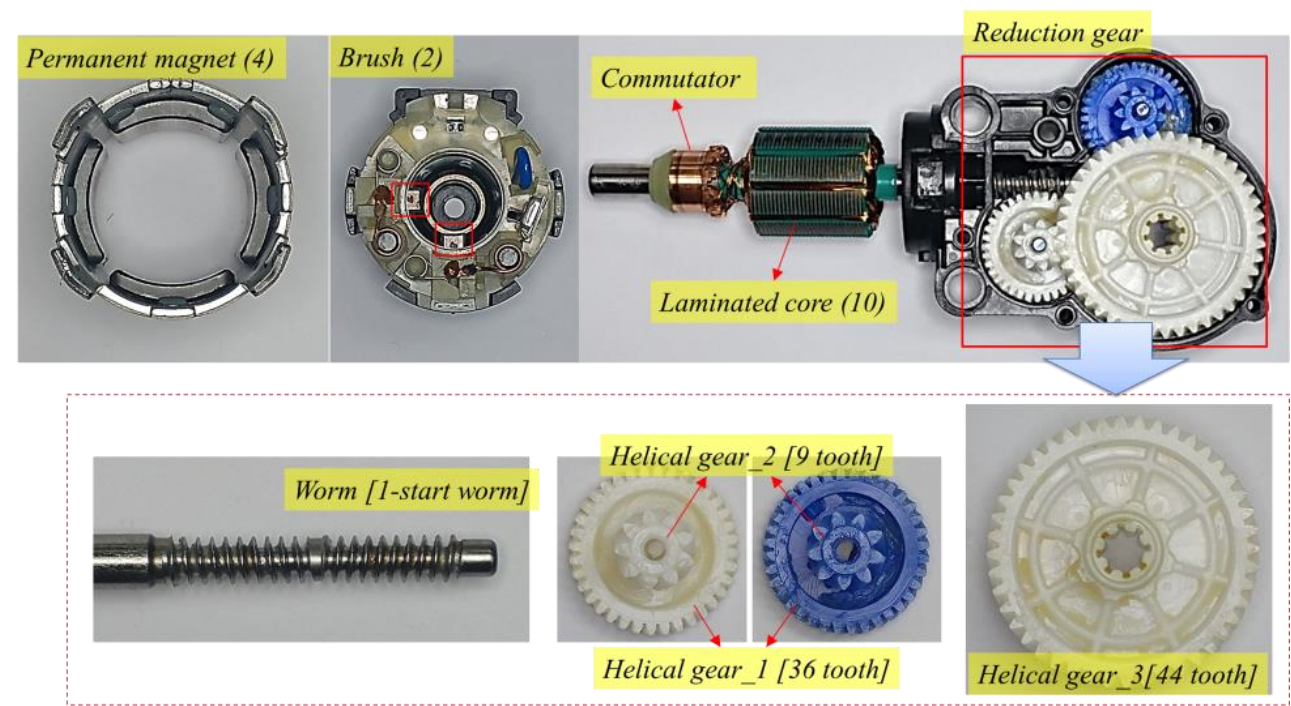

Fig. 3: Components of the DC Geared Motor.

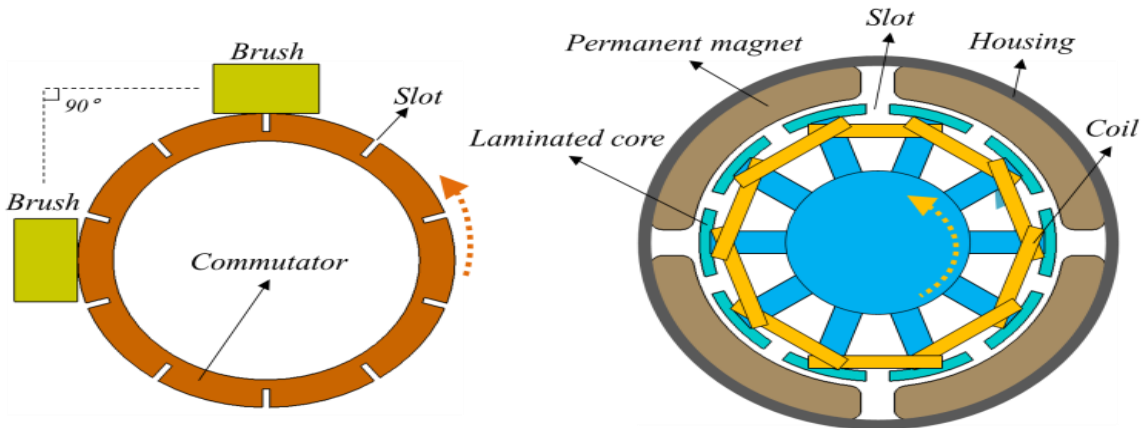

Fig. 4: Mechanism Schematic of Brush-Commutator and Permanent Magnet-Laminated Core.
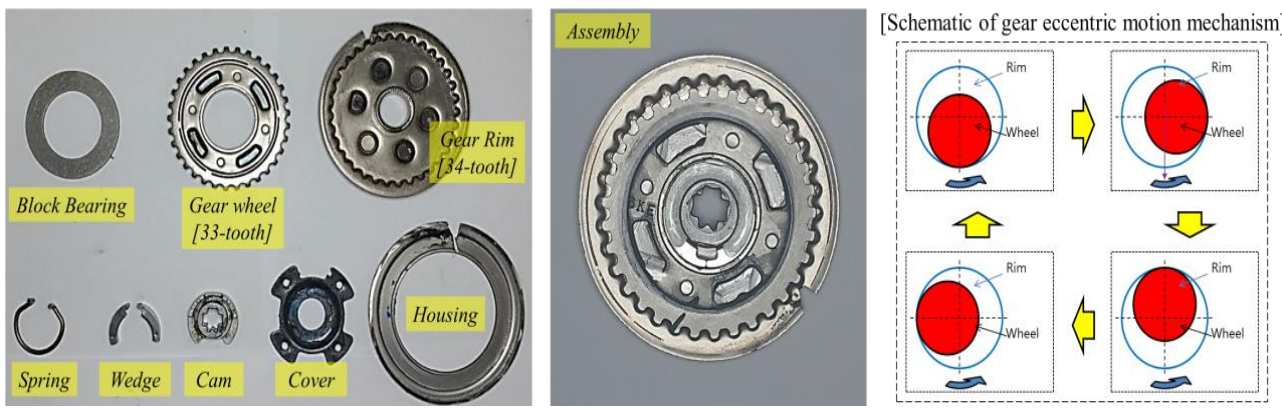

Fig. 5: Recliner Component and Mechanism Schematic.

Table 1: Frequency Components of DC Geared Motor and Recliner

\begin{tabular}{lll}
\hline [Average rpm: $3,960 \mathrm{rpm}]$ & Harmonic components of DC geared motor & Frequency $[\mathrm{Hz}]$ \\
\hline $\mathrm{f}_{\text {rpm }}$ (Average) & 1 st order & $66.00 \mathrm{~Hz}$ \\
$\mathrm{f}_{\text {brush }}$ & 20 th order & $1,320.00 \mathrm{~Hz}$ \\
$\mathrm{f}_{\text {torque }}$ & 20 th order & $1,320.00 \mathrm{~Hz}$ \\
$\mathrm{f}_{\text {motor, GMF }}$ & - & $16.50 \mathrm{~Hz}$ \\
$\mathrm{f}_{\text {recliner, GMF }}$ & - & $12.75 \mathrm{~Hz}$ \\
\hline
\end{tabular}

\subsection{Analysis of operating vibration}

The operating vibration of the backrest adjuster was analyzed based on the frequency components derived from Section 3.1. Figure 6 shows the color spectrogram for the vibration that occurred in each component. First, the 20th order ( $f_{\text {brush }}, f_{\text {torque }}$ ) was the highest in the DC geared motor, and $\mathrm{f}_{\mathrm{rpm}}$ was the next highest. Both recliners in the color spectrogram showed high levels at frecliner, GMF. In terms of the time data and the 1/3 octave band in Figure 7, the overall level of the right recliner was higher than that of the left recliner. It was judged that the right recliner was closer to the motor and thus greatly affected by it. Next the harmonic component of the motor appeared in the recliners, and this was also confirmed by the $1 / 3$ octave band. Lastly, it was confirmed that all the frequency components periodically fluctuated in the time domain. This was attributed to load change due to eccentric rotation of the recliner gear being caused by changing the rotation speed of the DC geared motor. 
(A) DC Geared Motor (Acc_1)

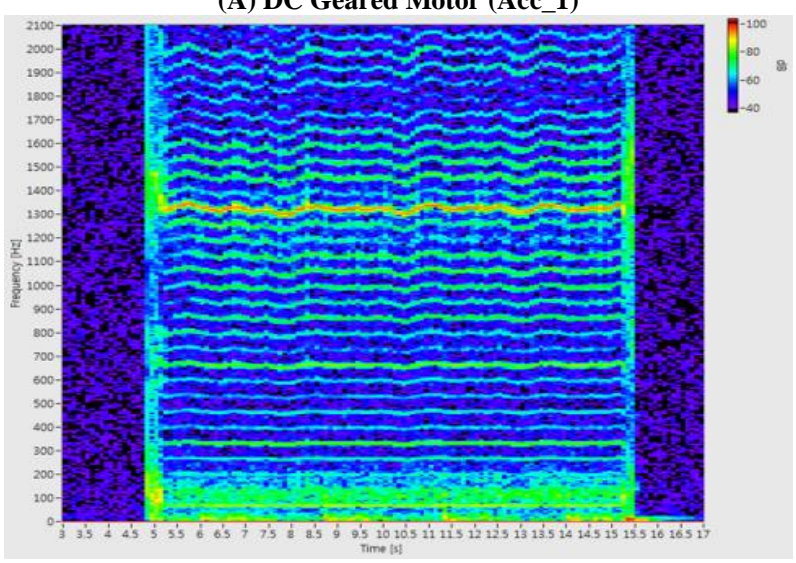

(B) Recliner_Left (Acc_2)

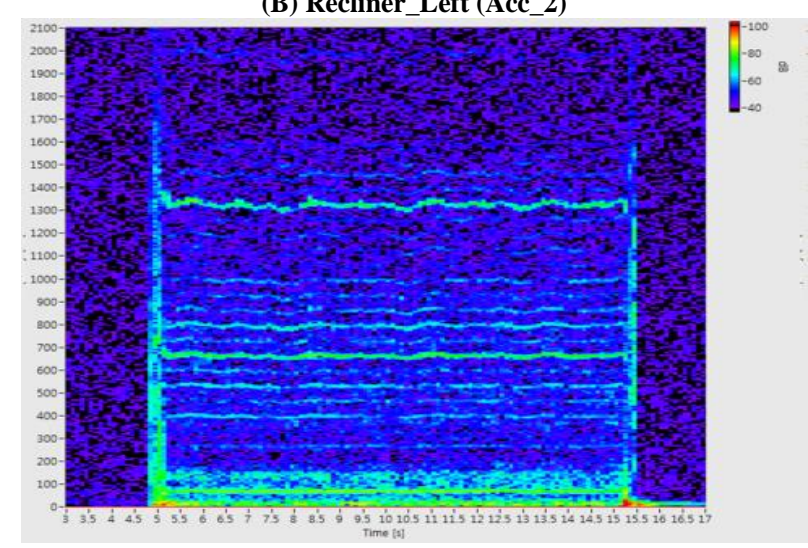

(C) Recliner_Right (Acc_3)

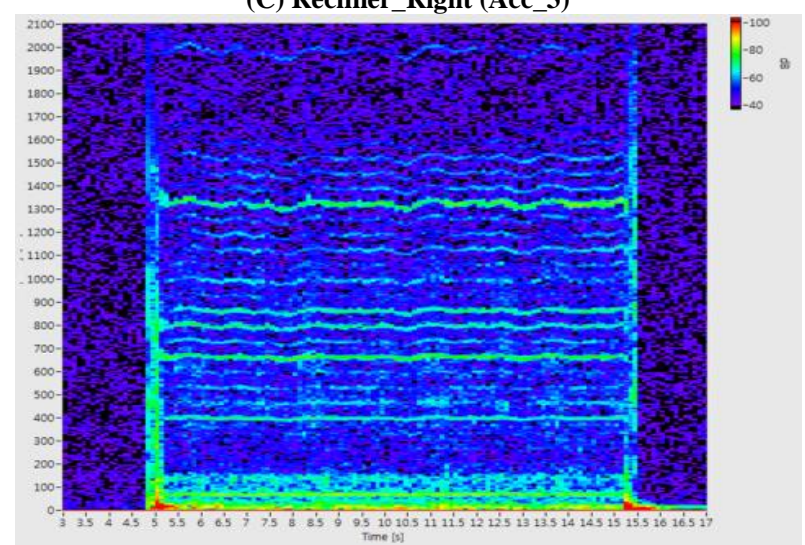

Fig. 6: Color Spectrogram for Operating Vibration of Each Component.

(A) Level Vs Time [Uncovered]

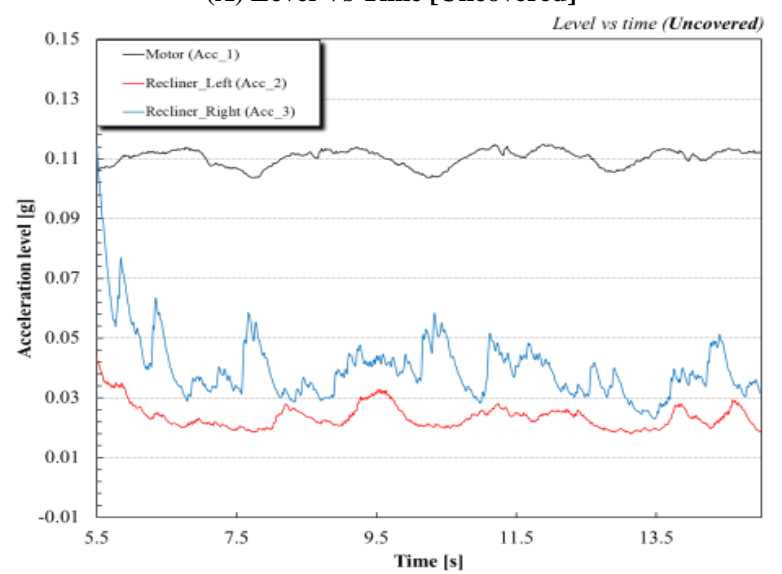

(B) 1/3 Octave Band [Uncovered]

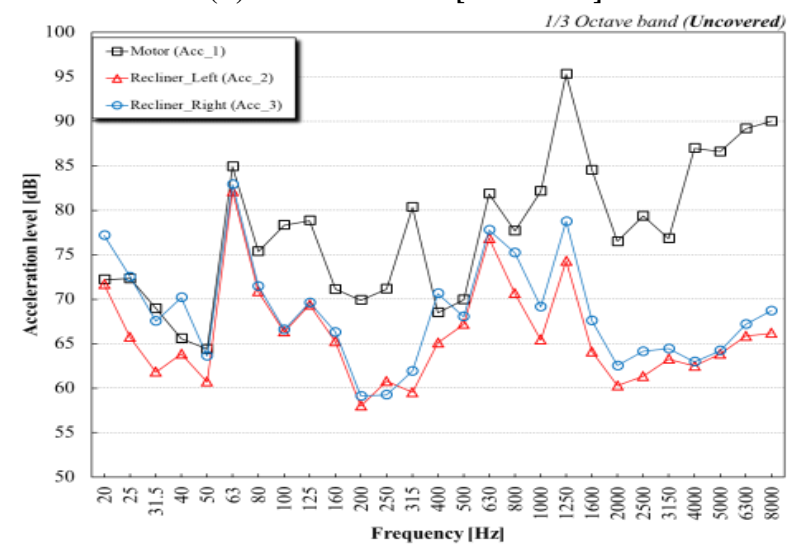

Fig. 7: Vibration Data of Each Component.

\subsection{Analysis of operating noise}

Based on Section 3.2, we analyzed the noise data. Figure 8 shows the color spectrogram for operating noise. The left figure represents the uncovered state and the right shows the pad-covered state. First, noise data characteristics in the uncovered state show that the 20th order of the DC geared motor was dominant, as was the case with the vibration data. In the case of $\mathrm{f}_{\mathrm{rpm}}$, vibration was slightly higher, but noise was not. In addition, there were somewhat higher levels of noise in the 10th and 30th order. This was evidence of a harmonic component generated between the $f_{r p m}$, fbrush and $f_{\text {torque }}$ components, which was predicted by the influence of the electromagnetic field and structure resonance of the motor. Next, the analysis result of the pad-covered state is as follows: The 20th order was again the highest, but the overall level and the 1/3 octave band in Figure 9 show that the overall noise level was significantly reduced. It was judged that the pad absorbed or blocked the harmonic noise of the motor. Also, by comparing the level of each component in the time data in Figure 9, this influence could be visually confirmed. Finally, based on the analysis so far, we analyzed the influence of the pad on the noise contribution of each component. This noise contribution analysis procedure was as follows: First, the sound pressure level corresponding to each frequency component was derived based on the center frequency of the 1/3 octave band. Here, the sound pressure level was in $\mathrm{dB}$, converted to physical unit pressure due to absence of linearity. Then the ratio of pressure to each component was calculated from the total pressure. Equation (4) defines the contribution $\left(\zeta_{\mathrm{c}, \mathrm{k}}\right)$ of the $1 / 3$ octave band component to the sound pressure of total noise.

$\zeta_{c, k}=\frac{P_{f_{c}}}{P_{t}} \times 100[\%]\left(\mathrm{P}_{\mathrm{t}}:\right.$ Total sound pressure, $\mathrm{P}_{\mathrm{fc}}$ : sound pressure of center frequency in $1 / 3$ octave band)

The result of the contribution is shown by the graph in Figure 10. In the uncovered state, the contribution of the 20th order was approximately $55 \%$ and the 30 th order was approximately $18 \%$. Together, these constituted about $73 \%$ of total sound pressure and their influence was dominant. On the other hand, in the pad-covered state, the 20th order contributed about $10 \%$ and all other frequency components comprised less than $10 \%$. When these were calculated as transmission loss, it was confirmed to be approximately $18 \mathrm{~dB}(\mathrm{~A})$ in the 20 th order and about $16 \mathrm{~dB}(\mathrm{~A})$ in the 30 th order. In conclusion, motor noise, which accounted for more than $70 \%$ of the backrest adjuster operating noise, caused considerable energy loss due to the influence of the pad. Of particular note, the noise reduction effect was deemed to amount to about $45 \%$ p for the 20th order, making it the most influential factor. 
(A) Uncovered

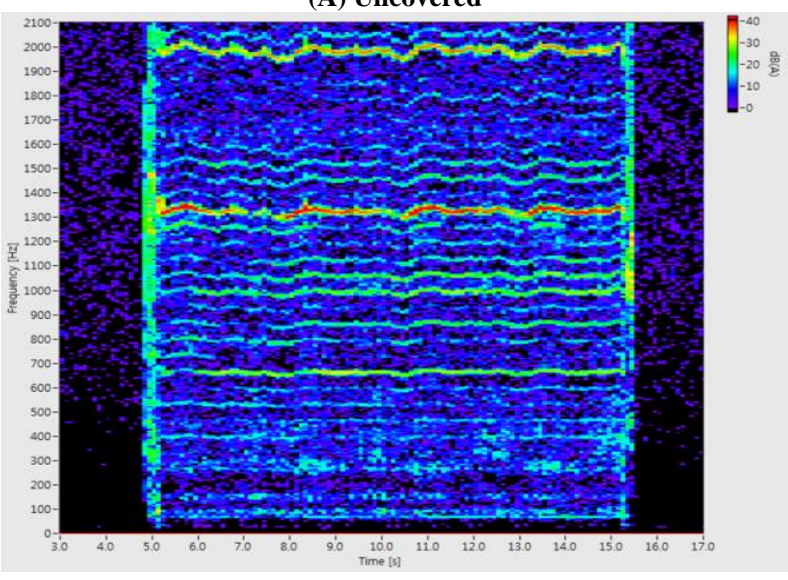

(B) Pad Covered

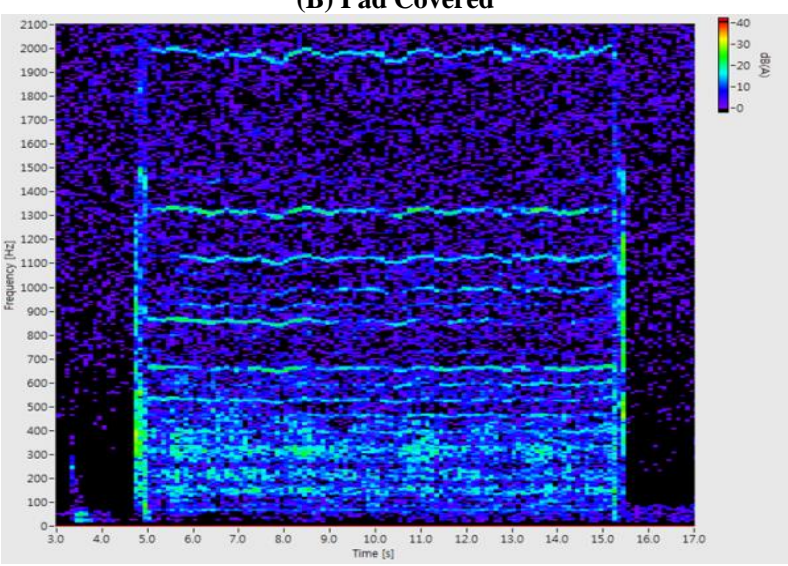

Fig. 8: Color Spectrogram for Operating Noise of Seat Back Rest.

(A) Level Vs Time [Uncovered]

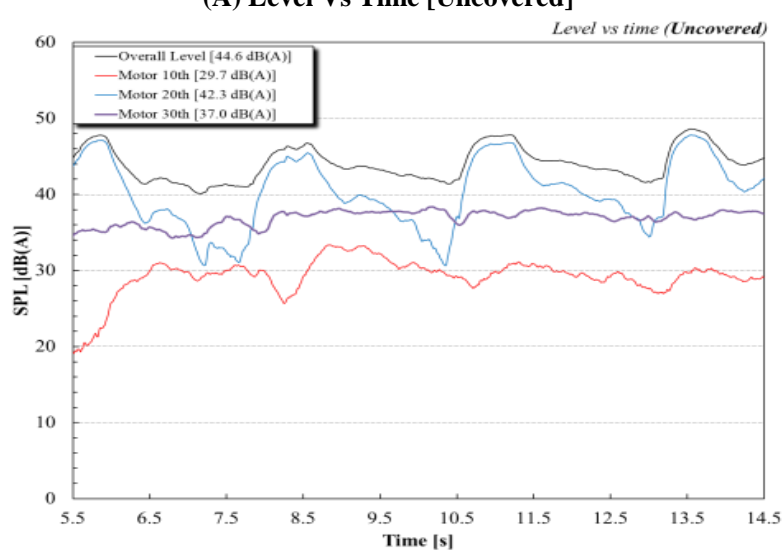

(B) Level Vs Time [Pad Covered]

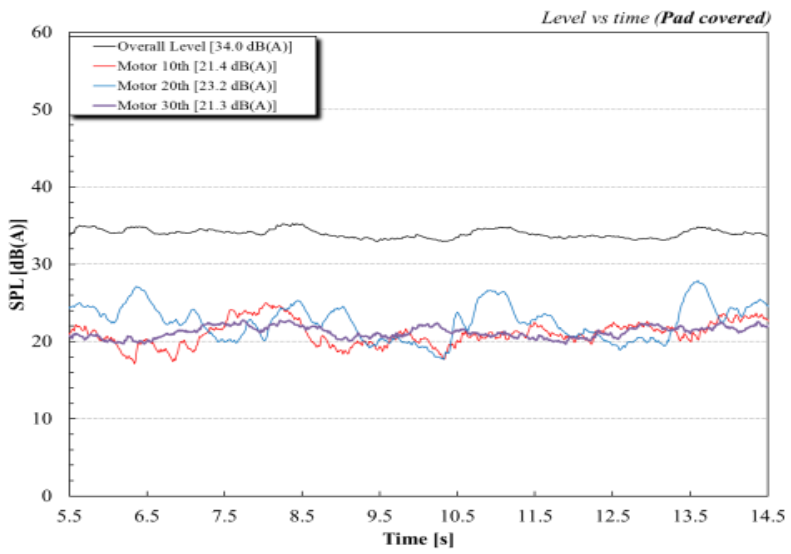

(C) 1/3 Octave Band

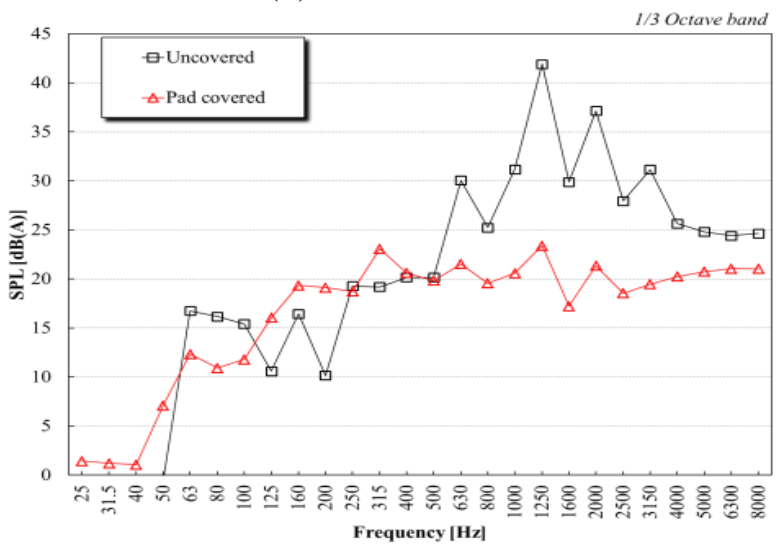

Fig. 9: Noise Data of Each Component.

(A) Noise Contribution

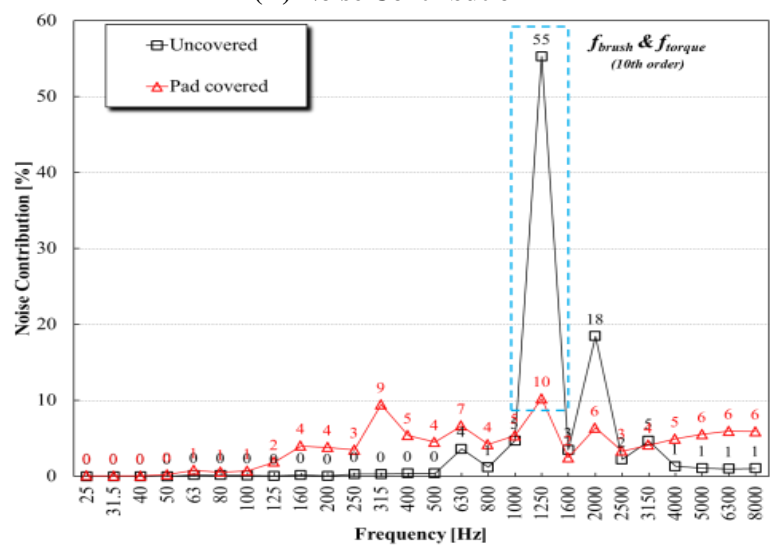

(B) Transmission Loss

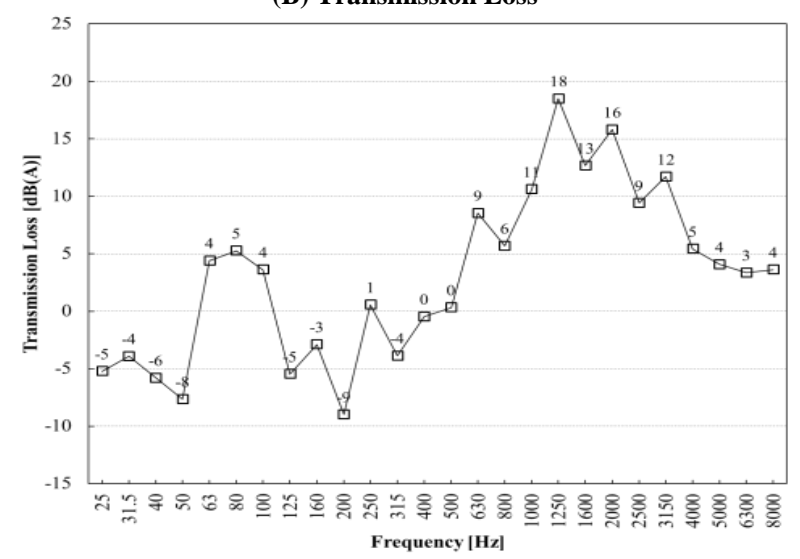

Fig. 10: Analysis of Noise Influence for Each Frequency Component.

\section{Conclusion}

In this study, we analyzed the respective roles played by noise source, contribution, and noise reduction effect on vehicle seat backrest adjuster operating noise. The conclusion was as follows.

1) The noise influence of the recliner was judged to be insignificant, but it was confirmed that the low frequency fluctuation caused by the total noise is dependent on change in the load generated by eccentric rotation of the recliner gear. This is because the load of the back frame is directly transmitted to the recliner gear. Therefore, if an error occurs in the gear teeth, a possibility exists of problems being caused such as abnormal gear chattering, unstable behavior, and noise increase in the DC geared motor due to the load. Therefore, it was found that the design of the recliner gear teeth was a decisive factor in controlling the motor load. 
2) The 20th order $(1,320 \mathrm{~Hz})$ of the DC geared motor was the main cause of operating noise, which was derived from friction noise of brush-commutator and torque ripple noise. This noise accounted for more than $55 \%$ of total operating noise. In addition, the 30th order amounted to approximately $18 \%$.In other words, the harmonic components of the DC geared motor created most of the noise, and changing the brush material and reducing torque ripple is expected to significantly improve the overall operating noise.

3) As a result of the seat pad noise reduction effect, total power decreased by more than $11 \mathrm{~dB}$ (A). In addition, it was confirmed that the 20th order cause of the main noise was reduced by about $45 \%$ p. However, since the properties of the seat pad are insufficient, employing a numerical approach to acoustic energy loss is difficult. Therefore, it is necessary to further investigate this noise reduction effect and acoustic energy loss in accordance with seat pad density and material.

\section{Acknowledgment}

This research was supported by Basic Science Research Program through the National Research Foundation of Korea (NRF) funded by the Ministry of Education (No. 2017R1D1A1B03033881) and Advanced Motor Parts RIC of Kongju National University administered by MOTIE (Ministry of Trade, Industry \& Energy), Korea.

\section{References}

[1] Kim, S. Y., An, J. R., \& Kim, K. S. (2016). A Study on the Stiffness Characteristics according to the Body Pressure on the Seat Cushion for Vehicle. Indian Journal of Science and Technology, 9(46), 1-6.

[2] Kim, S. Y., Kim, O. H., \& Kim, K. S. (2016). Analysis on the Stiffness of Automotive Seat Cushion based on Sitting Strategy of Vehicle Drivers. Journal of the Korean Society of Mechanical Technology, 18(6), 924-930.(http://www.earticle.net/article.aspx?sn=290885)

[3] Kim, S. Y., Jeon, O. H., \& Kim, K. S. (2017). A Study on the Abnormal Noise Characteristics for Moving Device of Automotive Power Seat. International Information Institute (Tokyo). Information, 20(5B), 3667-3674.

[4] Kim, S. Y., Jeon, O. H., \& Kim, K. S. (2016). A study on the experimental analysis of noise from vehicle power seat slide rail. International Journal of Control and Automation, 9(3), 133-142.

[5] Lee, D., Park, C., Lee, K., Kim, S., \& Kim, H. (2005). Evaluation of Static/Dynamic Structural Strength for Automotive Round Recliner. Transactions of the Korean Society of Automotive Engineers, 13(1), 140-146.

[6] Kim, S. Y., Lee, J. B., \& Kim, K. S. (2017). A Study on the Dynamic Analysis of Recliner Gear for Vehicle Power. Journal of the Korean Society of Manufacturing Process Engineers, 16(6), 15-20. (http://www.dbpia.co.kr/Article/NODE07282193)

[7] Kim, S. Y., \& Kim, K. S. (2018). A Study on Radiation Noise of Vehicle Power Seat Recliner using Finite Element Analysis. Journal of the Korean Society of Manufacturing Process Engineers, 17(1), 101-107. (http://www.dbpia.co.kr/Article/NODE07368134)

[8] Kwon, J. H., Kim, K. S., Lee, C. M., \& Hwang, S. M. (2009). Effect of the Number of Slots on the Acoustic Noise from BLDC Motors. Transactions of the Korean Society for Noise and Vibration Engineering, 19(8), 759-763.

[9] Jang, S. H., \& Jo, N. H. (2015). A Study on the Torque Ripple Reduction in Brushless DC Motors using Disturbance-Observer Controller. The Transactions of the Korean Institute of Electrical Engineers, 64(8), 1217-1223.

[10] GM Worldwide Engineering Standard, Test Procedure, Laboratory Evaluation of Power Seat Sounds, General Motors Company, GMW14240, 1-3. 